\title{
Fault Diagnosis of Coal Mining Machinery Based on State Parameters
}

\author{
Dong Song $^{1}$ \\ Taiyuan Research Institute of China Coal Technology \& Engineering Group, \\ Taiyuan, China
}

\begin{abstract}
The reliable operation of coal mining machinery acts as an important guarantee for safe productions in underground coal mines. The status monitoring and fault diagnosis of traditional coal mining machinery mainly rely on threshold judgments. However, a single judgment condition and a long fault propagation chain can be found in the method of threshold judgments, which make it difficult to accurately seek the fault type. By using the data analysis of state parameters for coal mining machinery, fault parameters and propagation paths can be analyzed effectively. This paper takes the cutting unit of a certain type of bolter miners as an example, a static and dynamic numerical analysis method of the cutting unit of bolter miners are established by virtue of FTA-Petri net models and BP-Firefly neural networks, which can provide a new perspective for fault diagnosis of coal mining machinery.
\end{abstract}

Keywords. FTA-Petri net models; BP-Firefly neural networks; fault diagnosis; cutting part

\section{Introduction}

Coal is the main source of energy in this day and age. Because the coal mine accidents happen frequently in some countries, how to improve the safety and reliability of coal mining machinery equipments becomes a universal attention question. The condition monitoring and fault diagnosis methods of traditional coal mining machinery are mainly based on on-line threshold judgments, which may be confronted with some limitations when using intelligent fault diagnosis methods. Currently, the online threshold method relies on the over-limit alarm based on motors and currents to judge the fault type. In addition, the online threshold fault diagnosis is usually a postjudgment method, which is based on local data analysis when the equipment is stopped. This method owns a single judgment condition and a long fault propagation chain, and it is difficult to accurately determine the fault type. What is worse, the maintenance cost is high by using the above-stated traditional methods [1-2]. By introducing the data analysis of state parameters for coal mining machinery, the failure propagation path can be effectively analyzed and the failure point can be accurately judged, which can enhance the performance of fault diagnosis of coal mining machinery from the aspect of costs, efficiencies, and accurate predictions [3-5].

\footnotetext{
${ }^{1}$ Corresponding Author: Dong Song, Taiyuan Research Institute of China Coal Technology \& Engineering Group, Taiyuan, China; Email: tymkysd@126.com
} 
Since coal mining equipments, such as bolter miners or roadheaders, act as complex heavy electromechanical systems, and the working environment and conditions of them are complicated and harsh. Therefore, intelligent fault diagnosis methods based on state parameters for coal mining machinery may face many challenges, such as insufficient detection approaches, more data interference factors, difficult data collections, etc. Nowadays, the fault diagnosis of coal mining machinery still relies on information threshold discrimination [6-8]. Recently, some scholars begin to use data mining-based methods to seek fault types for mining machinery such as artificial intelligence approaches including genetic algorithms and artificial neural networks, which allow employing the non-iterative methods, where one only needs to enter input parameters as the considered parameters of the process are generated as an answer to the input data set. Moreover, artificial neural networks have the ability to reproduce the process from training samples and specific knowledge of the process is not essential. Krzywanski et al. [9] introduced genetic algorithms and artificial neural networks approach for the optimization study of a hydrogen concentration in syngas via $\mathrm{CaO}$ Sorption. Yang et al. [10] established a fault tree along with a diagnosis expert system by establishing fuzzy expert systems to realize the on-line fault diagnosis and the fault interpretation of roadheaders.

In light of the above literature review, it is necessary to study data mining-based methods to seek fault types for mining machinery. In the current paper, we aim to obtain a satisfactory fault diagnosis conclusion by taking advantages of FTA-Petri net models and BP-Firefly neural networks within the background of bolter miners. To the best out knowledge, there are no reports on model research of fault diagnosis of bolter miners by using FTA-Petri net models and BP-Firefly neural networks. Thus, we first revisit some basic knowledge on FTA-Petri net models and BP-Firefly neural networks, then an experiment analysis is made to show the merit of the hybrid method.

\section{FTA-Petri net models}

Fault Tree Analysis (FTA) is an efficient data-mining method which is used to evaluate the safety and reliability for diverse systems. It mainly uses the causality diagram and takes the top event as the analysis object. Various hardware, software, environment and other factors are used as sub-events, and the relative logical relationship is determined according to the causality of each sub-event that leads to the occurrence of the top event. Then, the iterative calculation will stop until the basic event that causes the target event is found [11-12]. In what follows, we summarize the main notions of FTAPetri net models.

\section{(1) The occurrence probability of top events}

Suppose the failure probability of the bottom event $x_{i}$ is $q_{i}, n$ represents the number of bottom events, and the failure probability of minimum cut sets is provided as:

$$
P=P\left(x_{1} \mathrm{I} \quad x_{2} \mathrm{I} \quad \ldots \mathrm{I} \quad x_{m}\right)=\stackrel{m}{\mathrm{C}} q_{i},
$$

in the above formulae, $m$ represents the number of minimum cut sets. Let the minimum cut set be $F_{1}, F_{2}, \mathrm{~K}, F_{m}$, the occurrence probability of fault tree top events provided as: 


$$
P_{T}=\sum_{j=1}^{\mathrm{m}}\left(\left(\prod_{i \in K_{j}} F_{i}(t)\right),\right.
$$

the above formulae indicates the probability of top events is equal to the sum of the probability of minimum cut sets.

(2) Let $\sum=\left(P, T, F, K, W, Z, M_{0}\right)$ be a faulty petri net, $P=\left\{p_{1}, p_{2}, \ldots \ldots p_{m}\right\}$ and $T=\left\{t_{1}, t_{2} \ldots \ldots t_{n}\right\}$, then the structure of faulty petri nets can be represented by a matrix ( $m$ rows and $n$ columns) as follows:

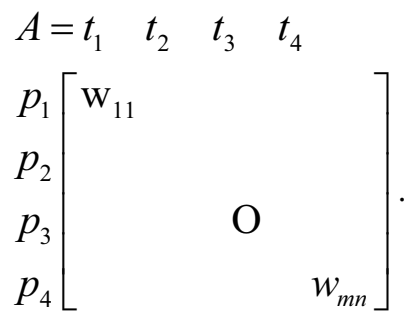

(3) The state equation of faulty Petri nets is

$$
M_{K}=M_{K-1}+A \oplus Z_{K},
$$

where $M_{K}$ and $M_{K-1}$ represent state identification sets of faulty networks at time $k$ and $k-1, A$ is the correlation matrix, $Z_{K}$ represents the sequence of the transition for faulty petri networks at time $k$, and the operation $\oplus$ represents the logical 'or' relationship.

\section{BP-Firefly neural networks}

The learning principle of BP neural networks involves the forward propagation of signals and the backward propagation of errors. After repeated iterative calculations, an artificial neural networks model is established to process nonlinear and unknowable complex information. In addition, BP neural networks mainly include an input layer, a hidden layer, and an output layer. The neurons in each layer are not connected, whereas the neurons in different layers are completely interconnected. Since BP neural networks own the limitations of slow convergence speed in learning processes, low robustness, and poor network performances, it is noted that the firefly algorithm can simulate cooperative group behaviors of fireflies' movings through lights and life habits such as foraging and mate selections. As a result, the firefly algorithm owns the advantage of simple structures, few adjustment parameters, and fast convergence speeds [13-15].

The motivation of using the firefly algorithm to optimize BP neural networks is listed below: we first use the firefly algorithm to train weights and thresholds of BP neural networks for solving the randomness of initial weights and threshold selections, that is, we make full use of the global optimization and heuristics optimization features of the firefly algorithm. Next, we use the BP neural network algorithm to get the final neural network structure. The fundamental steps of the optimization algorithm are shown as follows:

Step 1. Create a BP neural network, set network parameters, initialize weights and thresholds. 
Step 2. Set the parameters of the firefly algorithm, including the number of fireflies $n$, the light absorption intensity coefficient $\gamma$, the step size factor $\alpha$, the maximum attraction $\beta_{0}$, the maximum number of iterations $\max t$, and the adaptation threshold. Step 3. Randomly initialize all positions of firefly within a feasible region.

Step 4. Calculate the fitness value of the firefly, that is, calculate the objective function value. The process of BP network's training is that when the error value during iterative calculations, that is, when the mean square error is less than the set error threshold, the learning is considered to be completed, and the calculation is stopped, the result is the output. Since the firefly algorithm finds the maximum value, it is necessary to convert the minimum value into the maximum value. The fitness function is the reciprocal of the mean square error function of the BP neural network, that is, $f=\frac{1}{E}$, where $E=\frac{1}{N} \sum_{i=1}^{n}\left(D_{i}-Y_{i}\right)^{2}$. In the above formulae, $f$ is the objective function value, $E$ is the mean square error of the BP neural network, $N$ is the number of output nodes, $D_{i}$ is the target value of the $i$ th output node, and $Y_{i}$ is the actual value of the $i$ th output node.

Step 5. Calculate relative brightness and attractiveness, and determine the direction of movement. The relative fluorescent brightness of fireflies is $I=I_{0} e^{-\gamma r_{i j}}$. In the above formulae, $I_{0}$ is the maximum fluorescence brightness of the firefly, $\gamma$ is the light intensity absorption coefficient, and $r_{i j}$ is the spatial distance between the firefly $i$ and the firefly $j$. The attraction of fireflies is $\beta=\beta_{0} e^{-\gamma r_{i j}^{2}}$. In the above formulae, $\beta_{0}$ is the maximum attraction, that is, the attraction at the light source.

Step 6. Randomly disturb the firefly in the best position, and then update the position of the firefly. The position update formula for the firefly $i$ moving to the firefly $j$ because of its attraction is $x_{i}=x_{i}+\beta\left(x_{j}-x_{i}\right)+\alpha(\operatorname{rand}-1 / 2)$. In the above formulae, $\alpha$ is the step factor, rand is a uniformly distributed random number on $[0,1)$.

Step 7. Check whether the updated position of the firefly is out of the feasible range, and if it exceeds the range, use the boundary value as the updated position of the firefly.

Step 8. Increase the number of iterations by 1 to judge whether the termination condition is satisfied. The iteration is stopped if it is satisfied, and the weight and threshold are output. Otherwise, go to Step 4.

Step 9. Use the weights and thresholds output in Step 8 to train the neural network.

\section{Experimental analysis}

The cutting part of bolter miner mainly consists of cutting motor, cutting reducer, cutting drums, cutting lifting cylinders, sump cylinders and other devices. After analyzing common faults and their causes, a fault tree model of the cutting part for bolter miners is established, which is shown in Figure 1 as below. 


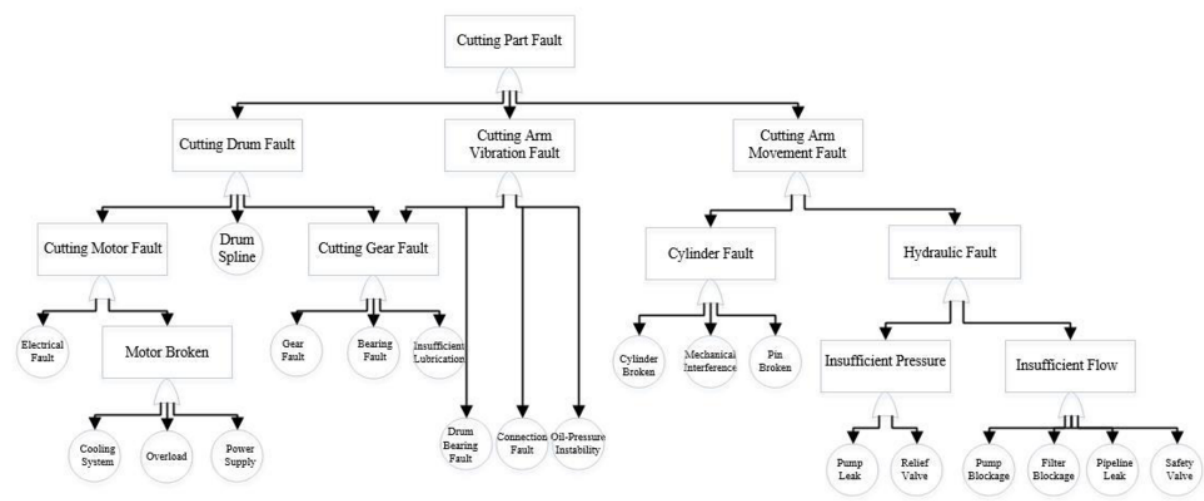

Figure 1. The fault tree model of cutting part of bolter miner

Due to the large scale of the fault tree model, the probability of occurrence of each fault can be judged based on expert scoring methods, and then the fault diagnosis can be performed through the top event probability calculation method. According to the weight of each fault event and the importance of each event, the optimal fault source search route is derived when the system owns problems, which provides a basis for fault diagnosis and saves time for fault diagnosis. However, the hierarchical analysis of the fault tree model is complex and the calculation process is lengthy, which make it difficult to perform quantitative analysis quickly. Based on FTA-Petri net models, the fault tree model can be greatly simplified, and hierarchical calculations can be transformed into matrix calculations. By establishing the faulty FTA-Petri net models, all the failure modes of bolter miners can be summed up.

Taking a cutting cylinder failure in a bolter miner in Shanxi Province, China as an example, the designed neural network adopts a three-layer structure, the number of input layer nodes is determined as 5, the number of output layer nodes is determined as 3 according to the features of a fault tree model of the cutting unit for bolter miners as shown in Figure 1. Moreover, we select the activation function as the Sigmoid function, the learning step length is set to 0.1 , the error factor is 0.0001 , the training function is traingd. After a trial-and-error procedure, we set the hidden neurons as 15 and the hidden layer as 1 . The parameters of the firefly BP neural networks are set as follows: the population number is 30 , the initial population of the firefly neural network is 30 , $\beta_{0}=1, \gamma=1$, and $\alpha=0.2$.

In the above-stated case, the input variables are symptoms of failures, and there are 5 in total. They are $x_{1}$ : temperature of the emission pipe; $x_{2}$ : presure of the scavenging box; $x_{3}$ : presure of the maximum breakout; $x_{4}$ : outlet temperature of the compressor rotor; $x_{5}$ : temperature of the scavenging box. The output variable is the cause of the failure, that is, the failure mode. There are 3 in total, namely $y_{1}$ : cylinder broken; $y_{2}$ : technical interface; $y_{3}$ : pin broken. There are 48 sets of training samples for the neural network, each with 12 sets of training samples for each type of fault. Some of the training samples are extracted and normalized as shown in Table 1. After the fault diagnosis procedure, we can obtain the output result as shown in Table 2. 
Table 1 The training samples

\begin{tabular}{|c|c|c|c|c|c|}
\hline \multirow{2}{*}{ Samples } & \multicolumn{5}{|c|}{ Input model (Fault symptom set) } \\
\hline & $x_{1}$ & $x_{2}$ & $x_{3}$ & $x_{4}$ & $x_{5}$ \\
\hline 1 & 0.568 & -0.702 & -0.752 & 0.065 & 0.088 \\
\hline 2 & 0.411 & 0.520 & 0.412 & 0.365 & -0.062 \\
\hline 3 & 0.236 & -0.185 & -0.396 & -0.366 & 0.114 \\
\hline
\end{tabular}

Table 2 The sample output of BP-Firefly neural networks

\begin{tabular}{|c|c|c|c|}
\hline \multirow{3}{*}{ Samples } & \multicolumn{3}{|c|}{ Diagnostic sample output } \\
\cline { 2 - 4 } & $y_{1}$ & $y_{2}$ & $y_{3}$ \\
\hline $\mathbf{1}$ & 1.0521 & -0.0121 & -0.1012 \\
\hline $\mathbf{2}$ & 0.6588 & 1.0358 & 0.0015 \\
\hline $\mathbf{3}$ & -0.3656 & 0.2565 & 0.8525 \\
\hline
\end{tabular}

It is easy to see from Table 2 that the fault types corresponding to sample 1 , sample 2 and sample 3 are $y_{1}, y_{2}$ and $y_{3}$ respectively.

In order to compare the performance of the network, 50 simulation tests were performed on the conventional BP neural networks and the BP neural network was optimized by the firefly algorithm. Under the same environment and parameter settings, the maximum and minimum average variances of the test sample network output are calculated respectively. The network performances are shown in Table 3.

Table 3 The network performances

\begin{tabular}{|l|r|r|c|}
\hline Training method & Average time/s & $\begin{array}{c}\text { Output maximum } \\
\text { average variance }\end{array}$ & $\begin{array}{c}\text { Output maximum } \\
\text { average variance }\end{array}$ \\
\hline conventional BP & 52.1251 & 0.0265 & 0.0025 \\
\hline BP-Firefly & 3.0235 & $9.52 \times 10^{-5}$ & $5.62 \times 10^{-7}$ \\
\hline
\end{tabular}

It can be seen from Table 3 that the BP neural network that is optimized by the firefly algorithm has higher accuracies in fault diagnosis of bolter miners and better network performances.

\section{Conclusions}

In this paper, the characteristic values that are selected from the cutting cylinder of bolter miners are used as the input vector of BP neural networks. By setting the neural network structure and initial parameters, the performance of the networks are tested, which can effectively explain the fault type of cutting cylinders of bolter miners in different working states. Through the establishment of bolter miner fault tree models, it can provide a guidance for quantitative analysis of the fault cause of bolter miners. The improved fault tree diagnosis method based on petri nets overcomes the conflicts, collisions, deadlocks and other phenomena of resources in traditional petri nets, and establishes theoretical reasoning process through matrix operations which provides a mathematical theoretical basis for the realization of intelligent diagnosis. In conclusion, FTA-Petri net models and the BP-Firefly algorithm provide new methods for fault diagnosis of coal mining machinery such as bolter miners and roadheaders. In the future, we can combine more convincing genetic algorithms to propose intelligent fault 
diagnosis rules. Moreover, we can also introduce granular-computing approaches, such as rough sets and three-way decisions, to explore related fault diagnosis approaches.

\section{Acknowledgements}

This work is supported by the Major Research and Development Project of Shanxi Province (No.201803D121121) and Science and Technology Innovation Project of China Coal Technology \& Engineering Group (No.2020-TD-ZD009).

\section{References}

[1] M. Bhuiyan, L. Parvez, M. Islam, et al. Heavy metal pollution of coal mine-affected agricultural soils in the northern part of Bangladesh. Journal of Hazardous Materials, 2010, 173(1-3): 384-392

[2] K. Ozgen, P. Warwick. Assessment of coal mine methane (CMM) and abandoned mine methane (AMM) resource potential of longwall mine panels: Example from Northern Appalachian Basin, USA. International Journal of Coal Geology, 2019, 208: 37-53

[3] C. Zhang, D. Li, Y. Mu, et al. A pythagorean fuzzy multigranulation probabilistic model for mine ventilator fault diagnosis. Complexity, 2018, 2018: 1-19

[4] C. Zhang, D. Li, J. Liang. Multi-granularity three-way decisions with adjustable hesitant fuzzy linguistic multigranulation decision-theoretic rough sets over two universes. Information Sciences, 2020, 507: 665-683

[5] L. Zou, Y. Li, F. Xu. An adversarial denoising convolutional neural network for fault diagnosis of rotating machinery under noisy environment and limited sample size case. Neurocomputing, 2020, 407: $105-120$

[6] Y. Hu, B. Ping, D. Zeng, et al. Research on fault diagnosis of coal mill system based on the simulated typical fault samples. Measurement, 2020, 161: 107864

[7] V. Agrawal, B. Panigrahi, P. Subbarao. Review of control and fault diagnosis methods applied to coal mills. Journal of Process Control, 2015, 32: 138-153

[8] X. Chen, W. Li, X. Yan. Analysis on rock burst danger when fully-mechanized caving coal face passed fault with deep mining. Safety Science, 2012, 50(4): 645-648

[9] J. Krzywanski, H. Fan, Y. Feng, et al. Genetic algorithms and neural networks in optimization of sorbent enhanced H2 production in FB and CFB gasifiers. Energy Conversion and Management, 2018, 171: 1651-1661

[10] B. Yang, D. Lim, A. Tan. VIBEX: an expert system for vibration fault diagnosis of rotating machinery using decision tree and decision table. Expert Systems with Applications, 2005, 28(4): 735-742

[11] M. Talebberrouane, F. Khan, Z. Lounis. Availability analysis of safety critical systems using advanced fault tree and stochastic Petri net formalisms. Journal of Loss Prevention in the Process Industries, 2016, 44: 193-203

[12] J. Wu, S. Yan, L. Xie. Reliability analysis method of a solar array by using fault tree analysis and fuzzy reasoning Petri net. Acta Astronautica, 2011, 69(11-12): 960-968

[13] B. Amiri, L. Hossain, J. Crawford, et al. Community detection in complex networks: Multi-objective enhanced firefly algorithm. Knowledge-Based Systems, 2013, 46: 1-11

[14] D. Biu, T. Nguyen, T. Ngo, et al. An artificial neural network (ANN) expert system enhanced with the electromagnetism-based firefly algorithm (EFA) for predicting the energy consumption in buildings. Energy, 2020, 190: 116370

[15] A. Hammid, M. Sulaiman, O. Awad. A robust firefly algorithm with backpropagation neural networks for solving hydrogeneration prediction. Electrical Engineering, 2018, 100: 3617-2633 